\title{
Neural frequency distributions may generate a new phase transition in models for synchronization
}

\author{
Marcelo HR Tragtenberg ${ }^{*}$, Caio L Tiedt, Mauricio Girardi-Schappo \\ From The Twenty Third Annual Computational Neuroscience Meeting: CNS*2014 \\ Québec City, Canada. 26-31 July 2014
}

Neural synchronization is a phenomenon related to information transmission between brain areas, cognitive functions, perceptual and motor skills and memory $[1,2]$, and also connected to mental illnesses like epilepsy, isolated seizures, Alzheimer's disease, Parkinson's disease, autism and schizophrenia [3]. This work is related to the critical state of the brain [4]: the order of the synchronization transition. We study this subject computationally using two models: Kuramoto's [5] and the KTz model [6].

The Kuramoto model is a paradigm in the study of synchronization. The order parameter for the synchronization exhibits a phase transition from a non-synchronized to a synchronized phase that can be first-order for a uniform distribution of the individual frequencies of the coupled oscillators, or second-order in the case of Lorentzian or Gaussian distributions [8]. The $\mathrm{KTz}$ is a formal neuron map-based model that can be coupled by map-based chemical synapses and also can exhibit synchronization phase transition under certain circumstances.

We study the transition between a uniform to a unimodal frequency distribution in the Kuramoto. This change in the frequency distribution is made through a particular kind of discretization of the Gaussian and Lorentzian distributions: we create distributions that are podia with $2 m+1$ steps. We study the cases from the uniform distribution $(m=0)$ to a continuous unimodal distribution $(m \rightarrow \infty)$. There is an abrupt transition in the behavior of the model as a function of $m$ : for $m>1$ the transition is always second order. We sum a noise term in the oscillator frequencies and in the interaction between oscillators, separately. For each of the two cases, the phase transition in the order parameter for the uniform distribution frequency seems to change the order from first to second.

We also have preliminary results for synchronization in the KTz model, studying an order parameter analog to that for the Kuramoto model [10]. The synchronization transition seems to be second-order as a function of the connection strength, as it should be to for a critical brain.

\section{Acknowledgement}

MGS acknowledges partial financial support from Brazilian agency CNPq.

Published: 21 July 2014

\section{References}

1. Uhlhaas P, Pipa G, Lima B, Melloni L, Neuenschwander S, Nikoli'c D, Singer W: Neural synchrony in cortical networks: history, concept and current status. Front Integr Neurosci 2009, 3:17.

2. Usher M, Schuster HG, Niebur E: Dynamics of populations of integrateand-fire neurons, partial synchronization and memory. Neural Comput 1993, 5:570-586.

3. Uhlhaas PJ, Singer W: Neural synchrony in brain disorders: relevance for cognitive dysfunctions and pathophysiology. Neuron 2006, 52:155-168.

4. Haimovici A, Tagliazucchi E, Balenzuela P, Chialvo DR: Brain organization into resting state networks emerges at criticality on a model of the human connectome. Phys Rev Lett 2013, 110:178101.

5. Acebrón JA, Bonilla LL, Pérez Vicente CJ, Ritort F, Spigler R: The Kuramoto model: a simple paradigm for synchronization phenomena. Rev Mod Phys 2005, 77:137-185.

6. Kuva SM, Lima GF, Kinouchi O, Tragtenberg MHR, da Silva ACR: A minimal model for excitable and bursting elements. Neurocomputing 2001, 38-40:255-261.

7. Ghosh A, Gupta S: Relaxation dynamics of the Kuramoto model with uniformly distributed natural frequencies. Physica A 2013, 392:3812-3818.

8. Batista CAS, Batista AM, de Pontes JAC, Viana RL, Lopes SR: Chaotic phase synchronization in scale-free networks of bursting neurons. Phys Rev $E$ 2007, 76:016218.

doi:10.1186/1471-2202-15-S1-P155

Cite this article as: Tragtenberg et al:: Neural frequency distributions may generate a new phase transition in models for synchronization. BMC Neuroscience 2014 15(Suppl 1):P155.

\footnotetext{
* Correspondence: marcelotragtenberg@gmail.com

Department of Physics, Federal University of Santa Catarina, 88040-900,

Florianópolis, SC, Brazil
} 